
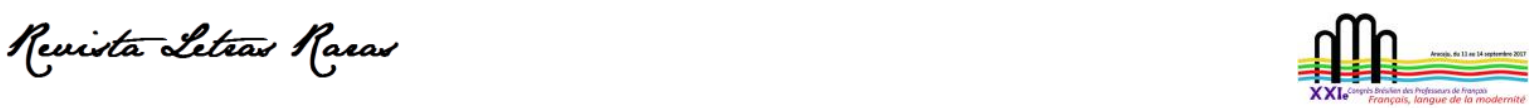

Actes du XXlème Congrès Brésilien des Professeurs De Français dans Édition spéciale de la Revue Letras Raras, 2021

ISSN : 2317-2347 - v. 10, Dossier Spécial (2021)

Todo o conteúdo da RLR está licenciado sob Creative Commons Atribuição 4.0 Internacional

\title{
Le français (langue et littérature) et l'idée de « modernité »
}

\author{
À la mémoire de Sabina Kundman
}

\begin{abstract}
Jean-Louis Chiss*
Sorbonne nouvelle (DILTEC)

Linguiste, spécialiste en épistémologie et en histoire des théories du langage et de la didactique des langues. II est aujourd'hui professeur émérite à l'Université Paris III (Sorbonne Nouvelle) où il a enseigné et a dirigé des thèses en Sciences du Langage et en Didactologie des Langues et des Cultures et a dirigé I'UFR (Unité de Formation et de Recherche) LLD (Littérature, Linguistique, Didactique). L'essentiel de son enseignement et de ses travaux de recherche porte sur l'enseignement/apprentissage du français langue maternelle, seconde et étrangère (didactique de la langue, des textes et discours), et sur l'histoire et l'épistémologie des sciences du langage (spécifiquement linguistique et grammaires françaises depuis la fin du XIXe siècle jusqu'à aujourd'hui).Ce parcours a été enrichi par la soutenance de plus de 25 thèses sous sa direction, de $8 \mathrm{HDR}$ (Habilitation à diriger de thèses) soutenues sous sa direction et la participation à une centaine de jurys de thèses et d'HDR. Auteur d'une cinquantaine d'articles, d'une vingtaine d'ouvrages, d'une quarantaine de chapitres d'ouvrage et d'une vingtaine de directions d'ouvrages, il a toujours participé fidèlement aux congrès nationaux, régionaux et mondiaux du réseau associatif des professeurs de français. J.-L. Chiss est un incontournable ami des professeurs brésiliens de français avec qui il partage son savoir, ses recherches et son amitié depuis 1996, lorsqu'il a commencé à diriger la formation des enseignants dans le cadre du projet La Bivalence : Didactique intégrée du portugais langue maternelle et $d u$ français langue étrangère. Depuis sa présence avec les conférences aux congrès brésiliens des professeurs de français est fidèle et très applaudie.
\end{abstract}

Reçu en : 10 août 2021. Approuvé en: 10 septembre 2021.

Comment citer cet article:

CHISS, Jean-Louis. Le français (langue et littérature) et l'idée de « modernité ». Revista Letras Raras, $p$. 188-193, n. Spécial, v. 10, nov. 2021.

En choisissant pour thématique « Le français langue de la modernité »"1, le congrès d'Aracaju nous invitait à méditer sur la relation entre une langue et un concept dont on sait l'inépuisable polysémie et le jeu de renvois, de croisements, de métamorphoses qu'il suscite.

\section{$\triangle \quad$ iean-louis.chiss@sorbonne-nouvelle.fr}

1 II faut de l'imagination pour concevoir des titres de congrès de professeurs de français associant cette langue à des notions toujours renouvelées (action, partage, ici modernité...) et suffisamment fédératrices pour que chacun y trouve sa place avec plus ou moins de bonheur. 

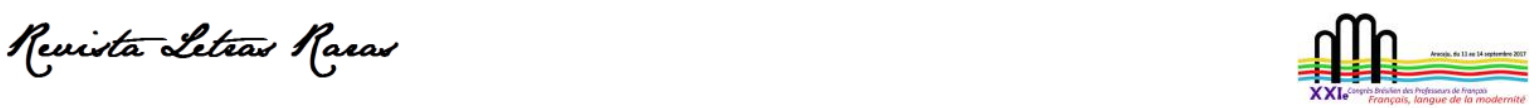

Actes du XXlème Congrès Brésilien des Professeurs De Français dans Édition spéciale de la Revue Letras Raras, 2021 ISSN : 2317-2347 - v. 10, Dossier Spécial (2021)

Todo o conteúdo da RLR está licenciado sob Creative Commons Atribuição 4.0 Internacional

\section{Variations sur la modernité}

Evidemment, on peut s'enquérir de la chronologie et des dichotomies qu'elle a fabriquées : au premier rang, l'opposition entre classique et moderne si vivace dans la culture française du langage mais avec des déplacements d'accent significatifs. A la fin du XVII ${ }^{\circ}$ siècle, la fameuse Querelle des Anciens et des Modernes est une polémique née au sein de l'Académie française et qui met face à face les partisans de l'imitation des grands auteurs de l'Antiquité (avec la révérence due aux modèles) et les sectateurs de l'innovation, plus attentifs à la création qu'à la reproduction fût-elle transformatrice. Mais le débat littéraire est aussi linguistique dans la mesure où il s'agit de continuer à affirmer la précellence de la langue française sur ses illustres devancières, le latin et le grec. II est intéressant de noter que parfois cette querelle est définie comme celle des Classiques et des Modernes dans un siècle précisément que la modernité ultérieure dénommera le siècle du classicisme. On en conclurait vite qu'on est toujours le moderne ou le classique de l'autre ${ }^{2}$...Mais si l'on poursuit en termes de périodisation, c'est le terme de contemporain qui s'imposera comme le tiers, après les deux premiers, qu'il s'agisse de la littérature ou de la langue. Et voici désormais l'extrême contemporain à propos des œuvres qui s'écrivent à partir des années $1980^{3}$.

Il est difficile avec le moderne de s'inscrire dans une temporalité précise ; certes nous connaissons la postmodernité comme nouvelle étape de la société occidentale mais ce postcorrespond plutôt à l'effacement des valeurs de la modernité, à la crise d'une philosophie des Lumières porteuse de progrès social et d'émancipation humaine, une forme de crise de la culture liée à l'effondrement des grands systèmes de pensée. Nous sommes passés de l'histoire à la philosophie. D'ailleurs, ces mêmes Lumières se sont accompagnées dès leur apparition au XVIII siècle d'un autre courant intellectuel porteur d'une conception réactive (on ne dit pas

\footnotetext{
2 Face à la culture classique, la fin du $\mathrm{XI} \mathrm{X}^{\circ}$ siècle voit apparaitre des humanités modernes comme filière de l'enseignement secondaire en France avec le recul du latin et l'installation des langues vivantes étrangères. Cette bipartition entre lettres classiques et lettres modernes reste vivace aujourd'hui : des deux côtés les lettres mais la question des langues fait la différence, les langues anciennes (latin d'abord mais aussi grec) marquent le classique quand le français associé à une langue étrangère signe le moderne. Remarquons que le classique se décline : les langues classiques synonyme des langues anciennes mais aussi la langue classique, les auteurs classiques, la littérature classique, dans des périodisations qui affectent une langue et une littérature (le latin classique, le bas latin, le latin tardif) et se retrouvent dans des organisations éducatives (les sections classiques vs les sections modernes).

${ }^{3}$ Voir par exemple Le roman français de l'extrême contemporain, sous la direction de B. Havercroft, P. Michelucci, P. Riendeau, Montréal, éditions Nota Bene, 2010.
} 
Actes du XXlème Congrès Brésilien des Professeurs De Français dans Édition spéciale de la Revue Letras Raras, 2021 ISSN : 2317-2347 - v. 10, Dossier Spécial (2021)

Todo o conteúdo da RLR está licenciado sob Creative Commons Atribuição 4.0 Internacional

« réactionnaire ») de la langue et de la littérature : «Les Antimodernes », de Joseph de Maistre à Roland Barthes, selon les bornes posées par Antoine Compagnon (2005), ont proposé une vision moins optimiste de l'histoire et de la société dans un style souvent où la nostalgie le dispute à l'indignation. On peut comprendre que, selon les points de vue, un auteur comme Baudelaire 4 puisse symboliser la pointe de la modernité ou figurer au rang des antimodernes. Sans doute, fautil saisir, avec ce même Baudelaire et ses grands lecteurs comme Walter Benjamin ou Henri Meschonnic, ce que porte la « modernité » en termes de contingence, d'énergie et de combat. A ce compte, la modernité n'est plus assignable à un fragment du temps : s'il y a une modernité du XIXe siècle, sans doute tout autant de l'antiquité : "La modernité caractérise une époque, elle caractérise en même temps l'énergie qui est à l'œuvre dans cette époque et qui la rapproche de l'antiquité » (Benjamin, 2002, p. 120).

Le mouvement qui conduit à transformer, par la littérature et l'art, la langue, la société et l'individu ne s'exprime pas nécessairement - loin s'en faut - dans un « contemporain [qui] ne cesse de courir après la modernité » (Meschonnic, 1993, p. 13), ce qui interroge, soit dit en passant, la modernité de l'extrême contemporain évoqué plus haut. Sans doute, les formes narratives de cette production romanesque signent-elles plutôt un retour qu'un changement, un consentement à l'époque plutôt qu'une avancée : « La modernité est un combat. Sans cesse recommençant. Parce qu'elle est un état naissant, indéfiniment naissant, du sujet, de son histoire, de son sens » (Meschonnic, 1993, p. 10).

Baudelaire, dans Le peintre de la vie moderne, partageait l'art entre les pôles du « fugitif », du « transitoire » et de l' " éternel », de l' « immuable » I| résistait à sa manière comme Rimbaud dont la fameuse phrase « II faut être absolument moderne » peut être interprétée comme l'injonction sociale devant laquelle le poète ne plie pas ou au contraire comme son vœu d'accéder à une modernité absolue.

\section{Des représentations de la langue française}

Nous sommes-nous éloignés des langues et du français? Sans doute pas de leurs représentations qui oscillent entre le vivant et le mort, le stable et le mouvant, entre la défense et

\footnotetext{
4 Dont Meschonnic écrit qu'il est « plus moderne que les post- et plus présent que jamais mais méconnaissable », Le français aujourd'hui $\mathrm{n}^{\circ}$ 92, 1990, p. 92.
} 

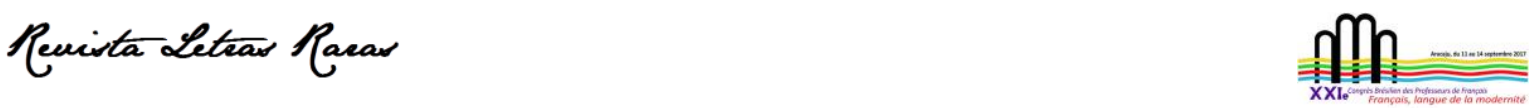

Actes du XXlème Congrès Brésilien des Professeurs De Français dans Édition spéciale de la Revue Letras Raras, 2021 ISSN : 2317-2347 - v. 10, Dossier Spécial (2021)

Todo o conteúdo da RLR está licenciado sob Creative Commons Atribuição 4.0 Internacional

l'offensive, entre le système et les discours. On connait l'idéologie linguistique (voir Chiss, 2018) qui, depuis le siècle classique, a cru devoir figer le français, vouloir exclure de le transformer, combattre toute innovation ou toute ouverture à la variation. Victor Hugo, dans la préface de Cromwell en 1827, fait tout à la fois œuvre de linguiste et de parangon de la modernité littéraire :

Les langues sont comme la mer : elles oscillent sans cesse. A certain temps, elles quittent un rivage du monde de la pensée et en envahissent un autre. Tout ce que leur flot déserté ainsi, sèche et s'efface du sol. C'est de cette façon que les idées s'éteignent et des mots s'en vont. II en est des idiomes comme de tout. Chaque siècle y apporte et emporte quelque chose. Qu'y faire ? Cela est fatal. C'est donc en vain que l'on voudrait pétrifier la mobile physionomie de notre idiome sous une forme donnée. C'est en vain que nos Josué littéraires crient à la langue de s'arrêter : les langues ni le soleil ne s'arrêtent plus. Le jour où elles se fixent c'est qu'elles meurent. Voilà pourquoi le français de certaines écoles contemporaines est une langue morte. (HUGO, 1985 [1827], p. 3031).

Le « génie de la langue française » s'est construit comme ensemble de représentations structurées à partir de sa « défense et illustration » (DU BELLAY, 1905 [1549])) dans un processus où les deux termes se sont longtemps mutuellement confortés et à un moment de concurrence avec le latin et d'autres langues comme l'italien : dans d'autres historicités, les rivaux ont été l'allemand puis l'anglais. Mais cette idée de « défense » d'une langue contre toutes les menaces du monde a fait son chemin, exercice solitaire dès lors que l'écrivain pouvait se considérer comme utilisateur, illustrateur d'une langue existant en dehors de lui. Que s'agit-il alors de défendre ? D'où l'offensive proustienne :

Les seules personnes qui défendent la langue française (comme l'armée pendant l'affaire Dreyfus), ce sont celles qui « l'attaquent ». Cette idée qu'il y a une langue française, existant en dehors des écrivains et qu'on protège est inouie. Chaque écrivain est obligé de se faire sa langue, comme chaque violoniste est obligé de faire son 'son'. (PROUST, 1936 [1908], p. 276).

L'offensive des écrivains s'inscrit contre la défense du pré carré de la langue, le protectionnisme linguistique qui garantirait l'exceptionnalité d'un français paré de toutes les vertus et mérites. C'est pourquoi, l'idéologie du génie se double, durant deux siècles au moins, d'une autre idéologie qui inverse les valeurs et transmue les qualités en défauts : de la « première » à la « dernière » des langues : « ...la langue française, sobre et timide, serait encore la dernière des langues si la masse de ses bons écrivains ne l'eût poussée au premier rang en forçant son naturel » (DE RIVAROL, 2013 [1784], p. 163-164). 

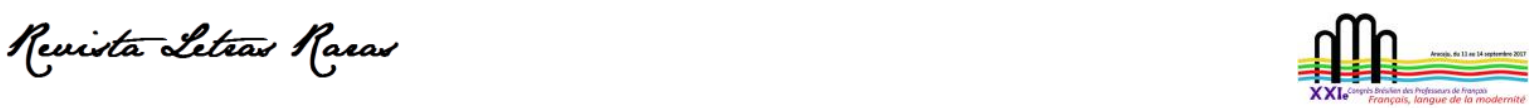

Actes du XXlème Congrès Brésilien des Professeurs De Français dans Édition spéciale de la Revue Letras Raras, 2021 ISSN : 2317-2347 - v. 10, Dossier Spécial (2021)

Todo o conteúdo da RLR está licenciado sob Creative Commons Atribuição 4.0 Internacional

Ce n'est que par les discours et les textes (dont la littérature) que les langues acquièrent, au-delà de leurs systèmes, leur caractère propre ou spécificité et que se pose, de manière pertinente, la question des valeurs comme celle de la modernité :

La langue française, comme les autres, peut exprimer à tout moment les valeurs les plus opposées. Mais sa chance singulière est que, pour la symboliser, le monde entier semble avoir choisi de ne retenir dans l'immense corpus des textes français que ceux qui étaient les plus riches d'avenir pour toute l'humanité : ceux qui posaient en termes inoubliables l'affirmation de l'universel. (ENCREVE ; BRAUDEAU, 2007, p. 33).

Certes, le réflexe des locuteurs, des professeurs, des organisateurs de congrès est d'attribuer aux langues les valeurs que portent les discours qui se sont déposés dans ces langues, ce que le grand linguiste et anthropologue Humboldt avait déjà clairement exprimé il y a deux siècles. II s'agit ici de faire signe en indexant le français sur la modernité. Prendre à rebours une image de la langue française empreinte de la patine du temps, lestée d'un patrimoine prestigieux devenu peut-être encombrant, liée aux salons de l'aristocratie et aux conversations mondaines, réservée aux élites culturelles. Pour lui substituer une autre image plus avenante, ouverte, démocratique, renvoyant à la jeunesse et à l'innovation, aux échanges commerciaux, à la technique, à la communication. C'est l'affaire du dépoussiérage. Mais une enquête historique démontrerait aisément que le français a toujours, dans le réel, répondu à tous ces réquisits. Le problème est qu'il en va ici des représentations où se pose moins la question de la vérité que celle des idées, des croyances, des imaginaires avec lesquels nous abordons le monde comme les langues. Alors oui, si l'on veut bien réinvestir dans modernité les dimensions précédemment évoquées - celles du mouvement, de l'intempestif, de la lutte, de l'historicité qui permet au passé de continuer à produire ses effets dans le présent - je me réjouis, tout en déconstruisant le syntagme langue de la modernité, qu'il ait pu nous réunir en ces jours de septembre 2017 à Aracaju.

\section{Références}

BENJAMIN, Walter. Charles Baudelaire. Un poète lyrique à l'apogée du capitalisme. Paris : Payot, 2002.

CHISS, Jean-Louis. La culture du langage et les idéologies linguistiques. Limoges : LambertLucas, 2018. 
Actes du XXlème Congrès Brésilien des Professeurs De Français dans Édition spéciale de la Revue Letras Raras, 2021 ISSN : 2317-2347 - v. 10, Dossier Spécial (2021)

Todo o conteúdo da RLR está licenciado sob Creative Commons Atribuição 4.0 Internacional

COMPAGNON, Antoine. Les Antimodernes de Joseph de Maistre à Roland Barthes. Paris: Gallimard, 2005.

DE RIVAROL, Antoine. Discours sur l'universalité de la langue française. Paris: Éditions Manucius, 2013 [1784].

DU BELLAY, Joachim. La défense et illustration de la langue française. Paris : Bibliothèque Internationale d'Édition E. Sansot \& Cie, 1905 [1549].

ENCREVE, Pierre ; BRAUDEAU, Michel. Conversations sur la langue française. Paris : Gallimard, 2007.

HUGO, Victor. Préface de Cromwell [1827] In : CEuvres complètes. Vol. 12. Paris : Robert Laffont, 1985, p. 1-44.

MESCHONNIC, Henri. Modernité, Modernité. Paris : Folio essais, 1993.

PROUST, Marcel. Lettre à Madame Straus, novembre 1908. In : Correspondance, VI. Paris : Plon, 1936, p. 276. 